\title{
A New Exact Solution of Burgers' Equation with Linearized Solution
}

\author{
Chun-Ku Kuo ${ }^{1,2}$ and Sen-Yung Lee ${ }^{1}$ \\ ${ }^{1}$ Department of Mechanical Engineering, National Cheng Kung University, Tainan 701, Taiwan \\ ${ }^{2}$ Department of Mechanical Engineering, Air Force Institute of Technology, Kangshan 820, Taiwan
}

Correspondence should be addressed to Sen-Yung Lee; sylee@mail.ncku.edu.tw

Received 24 March 2015; Accepted 13 August 2015

Academic Editor: Rosana Rodriguez-Lopez

Copyright (C) 2015 C.-K. Kuo and S.-Y. Lee. This is an open access article distributed under the Creative Commons Attribution License, which permits unrestricted use, distribution, and reproduction in any medium, provided the original work is properly cited.

\begin{abstract}
This paper considers a general Burgers' equation with the nonlinear term coefficient being an arbitrary constant. Two identical solutions of the general Burgers' equation are separately derived by a direct integration method and the simplest equation method with the Bernoulli equation being the simplest equation. The proposed exact solutions overcome the long existing problem of discontinuity and can be successfully reduced to linearity, while the nonlinear term coefficient approaches zero. In addition, a general Cole-Hopf transform is introduced. Finally, the proposed derived solution is compared with the perturbation solution and other existing exact solutions. A new phenomenon, which we named "kink sliding," is observed.
\end{abstract}

\section{Introduction}

Burgers' equation was first introduced by Bateman [1] in 1915 and later analyzed by Burgers [2] in 1948. The equation is used as a model in many fields such as acoustics [3], continuous stochastic processes [4], dispersive water [5], shock waves [5], heat conduction [6], and turbulence [7]. Burgers' equation can also be considered as a simplified form of the Navier-Stokes equation [8] due to the form of the nonlinear convection term and the occurrence of a viscosity term.

Burgers' equation is one of the very few nonlinear partial differential equations that can be solved exactly. When the absolute value of the nonlinear term coefficient of Burgers' equation is 2 or 1 , the exact solutions can be derived by the modified extended tanh-function method [9], the Expfunction method [10], the tanh-coth method [11], and the Cole-Hopf transform method [11-16]. In this paper, the nonlinear term coefficient of Burgers' equation is considered an arbitrary constant. The exact solutions of thisgeneral Burgers' equation are derived by the four methods mentioned above [9-16]. In addition, they are also separately derived by the simplest equation method with the Riccati equation being the simplest equation and a newly developed general ColeHopf transform method [11, 12]. It is shown that when the coefficient of the nonlinear term is the same as the regular Burgers' equation, the derived exact solutions are the same as those in the existing literature. However, all these exact solutions do not satisfy the continuity condition and will not be reducible to linear solutions when the coefficient of the nonlinear term in the Burgers' equation approaches zero.

In this paper, new exact solutions of the general Burgers' equation are separately derived by a direct integration method and the simplest equation method with the Bernoulli equation being the simplest equation, respectively. The two exact solutions are shown to be the same. In addition, the newly derived solution can be successfully reduced to linearity, while the nonlinear term coefficient approaches zero. Finally, the proposed derived solution is compared with the perturbation solution and other existing exact solutions. Several numerical results are presented and illustrated.

\section{General Burgers' Equation}

Consider the general Burgers' equation:

$$
u_{t}+\alpha u u_{x}-v u_{x x}=0 \text {, }
$$


where the nonlinear term coefficient $\alpha$ is an arbitrary constant and $v$ is the coefficient of the kinematics viscosity of fluids and is equal to $1 / R$. Further, $R$ is the Reynolds number, and when it is large the equation describes shock wave behavior [3].

2.1. Existing Exact Solutions. Following the solution methods and procedures of the modified extended tanh-function method [9], the Exp-function method [10], and the tanhcoth method [11], the following sets of solutions have been obtained.

One set, when $c>0$, is a front wave (kink) solution,

$$
u(x, t)=\frac{c}{\alpha}-\frac{c}{\alpha} \tanh \left(\frac{c}{2 v}(x-c t)\right),
$$

while the other is a traveling wave solution,

$$
u(x, t)=\frac{c}{\alpha}-\frac{c}{\alpha} \operatorname{coth}\left(\frac{c}{2 v}(x-c t)\right) .
$$

For $c<0$, the two traveling wave solutions are

$$
\begin{aligned}
& u(x, t)=\frac{c}{\alpha}+\frac{c}{\alpha} \tan \left(\frac{c}{2 v}(x-c t)\right), \\
& u(x, t)=\frac{c}{\alpha}-\frac{c}{\alpha} \cot \left(\frac{c}{2 v}(x-c t)\right) .
\end{aligned}
$$

Here, $c$ is the wave speed. When $\alpha=1$, solution (2) is the same as that obtained by Ebaid [10]; however, when $\alpha=-2, v=1$, solutions (2) to (5) are the same as those obtained by Wazwaz [11]. Many other solutions can also be found in Wazwaz's book [12].

2.2. The Simplest Equation Method with Riccati Equation being the Simplest Equation. The simplest equation method with Riccati equation being the simplest equation is a method commonly used to develop the exact solution of some nonlinear equations. It can also be used to develop exact solutions of the general Burgers' equation.

Herein, a new variable is introduced, $\xi=x-c t$, so that $U(\xi)=u(x, t)$. General Burgers' equation (1) is thus transformed into

$$
-c U_{\xi}+\alpha U U_{\xi}-\nu U_{\xi \xi}=0
$$

To solve the problem by the simplest equation method [17, 18 ], the solution can be assumed to be in the form of

$$
U(\xi)=\sum_{i=0}^{L} a_{i}[Y(\xi)]^{i}
$$

where $a_{i}$ are constants to be determined; $Y$ is the exact solution of a chosen nonlinear ordinary differential equation, called the simplest equation; and $L$ is a constant to be determined and the power of the specified solution function finite series, $Y$.

Now, consider the following Riccati equation as the simplest equation:

$$
Y_{\xi}=\mu\left(1-Y^{2}\right)
$$

where $\mu$ is wave number and $\mu>0$. The exact solution for the chosen Riccati equation (8) is

$$
Y=\tanh (\mu \xi)
$$

To determine the parameters, substitute (7) and (8) back into (6) and balance the linear terms of the derivative's highest order, which yields $U_{\xi \xi}$ with the highest order nonlinear term $U U_{\xi}$ in (6). Setting coefficients of like power of $Y$ to be zeros yields the following equations:

$$
\begin{aligned}
L+2(2-1) & =L+L+(2-1), \\
Y^{0}:-c+a_{0} \alpha & =0, \\
Y^{1}: a_{1} \alpha+2 \nu \mu & =0, \\
Y^{2}:-c-a_{0} \alpha & =0, \\
Y^{3}:-a_{1} \alpha-2 \nu \mu & =0 .
\end{aligned}
$$

The equations give $L=1$ and $a_{0}=c / \alpha, a_{1}=-2 \nu \mu / \alpha$. Consequently, the exact solution of the Burgers' equation is obtained:

$$
u=\frac{c}{\alpha}-\frac{2 \nu \mu}{\alpha} \tanh (\mu(x-c t))
$$

If one sets $\mu=c / 2 \nu$, then $c>0$. It can accordingly be found that solution (11) is exactly the same as that given in (2).

2.3. General Cole-Hopf Transformation Solution. When $\alpha=$ -2 and $\alpha=-1$, regular Cole-Hopf transformations, $u=$ $\nu(\partial / \partial x) \ln F$, and $u=2 \nu(\partial / \partial x) \ln F$ were introduced to study the associated Burgers' equations $[11,12,15]$, respectively. In this paper, a general Cole-Hopf transformation solution is developed to derive an exact solution of the general Burgers' equation.

The regular Cole-Hopf transformation in the following form is first modified:

$$
u=-\beta \nu \frac{\partial}{\partial x} \ln F
$$

where $\beta$ is a constant to be specified. Substituting (12) into (1) yields

$$
\begin{aligned}
& -\beta \nu \frac{\partial}{\partial x} \frac{F_{t}}{F} \\
& +\beta v^{2}\left(\frac{F_{x x} F_{x}}{F^{2}}(\alpha \beta-3)+\left(\frac{F_{x}}{F}\right)^{3}(2-\alpha \beta)+\frac{F_{x x x} F}{F^{2}}\right)
\end{aligned}
$$

$=0$.

Obviously, if $\alpha \beta=2$, (13) can be reduced to

$$
\begin{gathered}
-\beta \nu \frac{\partial}{\partial x} \frac{F_{t}}{F}+\beta v^{2}\left(\frac{-F_{x x} F_{x}}{F^{2}}+\frac{F_{x x x} F}{F^{2}}\right) \\
=\beta \nu \frac{\partial}{\partial x}\left(\frac{F_{t}-v F_{x x}}{F}\right)=0 .
\end{gathered}
$$


Consequently, the following linear equation is generated:

$$
F_{t}-\nu F_{x x}=0 .
$$

Moreover, it is a heat conduction equation with a solution in the form of $[11,12]$

$$
F=1+e^{k_{1} x+\nu k_{1}^{2} t}
$$

where $k_{1}$ is an arbitrary constant.

Therefore, the general Cole-Hopf transformation for the Burgers' equation is

$$
u=-\frac{2 v}{\alpha} \frac{\partial}{\partial x} \ln F
$$

and an exact solution of the general Burgers' equation can be derived as

$$
u=\frac{-2 v}{\alpha} \frac{k_{1} e^{k_{1} x+v k_{1}^{2} t}}{1+e^{k_{1} x+v k_{1}^{2} t}} .
$$

From the solutions above, it can be observed that the tanh-coth solutions, (2)-(5), the Cole-Hopf solution, (18), the solution derived from the simplest equation method with the Riccati equation being the simplest equation, and (11) are all proportional to $1 / \alpha$. In particular, when $\nu=1$, the Cole-Hopf solution, namely (18), with $k_{1}=-1$ is the same as one of the tanh-coth solutions, namely (2), with $c=1$.

It is well known that, for solution continuity, if the nonlinear coefficient $\alpha$ in a nonlinear differential equation (1) approaches zero, the equation is reduced to a linear one and the nonlinear solution should be reducible to a linear solution. However, from the existing exact solutions above, it can be observed that when the nonlinear coefficient $\alpha$ is reduced to zero, the solutions become infinite. Therefore, none will satisfy the solution continuity condition. In the following, a new exact solution of the general Burgers' equation with a linearized solution is developed.

\section{New Exact Solution}

3.1. Direct Integration Method. Integrating (6) once with respect to $\xi$ and setting the integration constant to be zero yields

$$
\nu U_{\xi}-\frac{\alpha}{2} U^{2}+c U=0 .
$$

Equation (19) can then be expressed as

$$
U_{\xi}=U(p+q U)
$$

where $p=-c / v, q=\alpha / 2 \nu$.

Integrating (20) once with respect to $\xi$ and using the result given in the integration table [19], it becomes

$$
\frac{-1}{p} \log \frac{p+q U}{U}=\xi+\xi_{0} \text {. }
$$

Therefore

$$
U=\frac{p e^{p\left(\xi+\xi_{0}\right)}}{1-q e^{p\left(\xi+\xi_{0}\right)}}
$$

where $\xi_{0}$ is an integral constant. As a result, a new exact solution of the general Burgers' equation can be derived as

$$
u(x, t)=\frac{-2 c}{2 v e^{(c / v)\left(x-c t+\xi_{0}\right)}-\alpha} .
$$

When the nonlinear coefficient $\alpha$ in nonlinear differential equation (1) approaches zero, the exact solution, namely (23), is reduced to

$$
u(x, t)=\frac{-c}{v} e^{(-c / v)\left(x-c t+\xi_{0}\right)} .
$$

This solution is consistent with the exact solution of the following linearized equation from Burgers' equation:

$$
u_{t}-v u_{x x}=0
$$

3.2. The Simplest Equation Method with the Bernoulli Equation being the Simplest Equation. Following the simplest equation method as discussed in Section 2.2, the same new exact solution can also be obtained by employing the simplest equation method with the following Bernoulli equation being the simplest equation [20-22]:

$$
Y_{\xi}=a Y+b Y^{2}
$$

Equation (26) has the exact solution in the form of

$$
Y=\frac{a e^{a\left(\xi+\xi_{0}\right)}}{1-b e^{a\left(\xi+\xi_{0}\right)}}
$$

After substituting (7) and (26) back into (6) and balancing the linear terms of the derivative's highest order, $U_{\xi \xi}$, with the highest order nonlinear term $U U_{\xi}$ in (6), $L=1$, and the following equations are obtained

$$
\begin{aligned}
Y^{1}:-c a_{1} a+\alpha a_{0} a_{1} a-v a_{1} a^{2} & =0, \\
Y^{2}:-c a_{1} b+\alpha a_{0} a_{1}+\alpha a_{1}^{2} a-3 v a_{1} a b & =0, \\
Y^{3}: \alpha a_{1}^{2} b-2 v a_{1} b^{2} & =0 .
\end{aligned}
$$

These equations give $a_{0}=0, a_{1}=1$, and $a=-c / \nu, b=$ $\alpha / 2 \nu$. Consequently, the solution is derived as

$$
U(\xi)=a_{0}+a_{1} Y=\frac{(-c / \nu) e^{(-c / \nu)\left(\xi+\xi_{0}\right)}}{1-(\alpha / 2 \nu) e^{(-c / v)\left(\xi+\xi_{0}\right)}} .
$$

In terms of $x$ and $t$ variables, the new exact solution of the general Burgers' equation can be expressed as

$$
u(x, t)=\frac{(-c / \nu) e^{(-c / v)\left(x-c t+\xi_{0}\right)}}{1-(\alpha / 2 \nu) e^{(-c / v)\left(x-c t+\xi_{0}\right)}} .
$$

After multiplying the numerator and denominator of (30) by $2 v e^{(c / v)\left(x-c t+\xi_{0}\right)}$, the same solution as that given in $(23)$ is obtained. 
3.3. The Perturbation Solution. The perturbation method is commonly used to study nonlinear equations and obtain approximate analytic solutions. The transformed general Burgers' equation, (6), can be set into the form

$$
-c U_{\xi}+\varepsilon U U_{\xi}-\nu U_{\xi \xi}=0
$$

where $\varepsilon=\alpha$ is a perturbation term. The perturbation solution is assumed to be in the following form:

$$
U=U_{0}+\varepsilon U_{1}+\varepsilon^{2} U_{2}+\cdots
$$

Substituting (32) into (31) and setting like power coefficients of $\varepsilon$ to be zeros, the following equations are obtained:

$$
\begin{aligned}
& \varepsilon^{0}: c U_{0 \xi}+\nu U_{0 \xi \xi}=0, \\
& \varepsilon^{1}: c U_{1 \xi}+\nu U_{1 \xi \xi}=U_{0} U_{0 \xi}, \\
& \varepsilon^{2}: c U_{2 \xi}+\nu U_{2 \xi \xi}=U_{0} U_{1 \xi}+U_{1} U_{0 \xi} .
\end{aligned}
$$

The approximated solution is

$$
\begin{aligned}
U= & -C_{1} \frac{c}{\nu} e^{(-c / v)\left(\xi+\xi_{0}\right)} \\
& +\varepsilon\left(\frac{-c}{v} e^{(-c / v)\left(\xi+\xi_{0}\right)}+\frac{1}{2 c} e^{(-2 c / v)\left(\xi+\xi_{0}\right)}\right) \\
& +\varepsilon^{2}\left(\frac{-c}{v} e^{(-c / v)\left(\xi+\xi_{0}\right)}+\frac{1}{4 c^{2}} e^{(-3 c / v)\left(\xi+\xi_{0}\right)}\right)+\cdots,
\end{aligned}
$$

where $C_{1}$ is a constant. When the coefficient of the perturbation term $\varepsilon$ is zero, (31) is reduced to a common linear differential equation. Solution (34) is reduced to being linear and is the same as the solution reduced from (24).

\section{Numerical Results and Discussions}

To illustrate the analysis, several numerical results are presented and illustrated.

Figure 1 illustrates the difference between the new solution and the perturbation solution, with $v=1$ and $c=1$, at $\xi=0$, as the nonlinear term coefficient $\alpha(\varepsilon)$ varies from -1 to 1 . For comparison, the constant $\xi_{0}$ in (23) and (34) is set to zero. It can be found that when $\alpha=0$, both solutions lead to linear solutions and are the same; however, when the absolute value of $\alpha$ is increased to one, the perturbation solution will have significant errors. Figure 2 shows the difference between the new exact solution and the perturbation solution with the absolute value of $\alpha$ being one, $v=1$ and $c=1$, at various $\xi$. It can be observed that the difference between the two solutions becomes negligible when $\xi$ is large $(\xi>4)$.

A kink wave is defined as a traveling wave which (1) rises or descends from one asymptotic state to another and (2) approaches a constant at infinity [12]. Moreover, when $x$

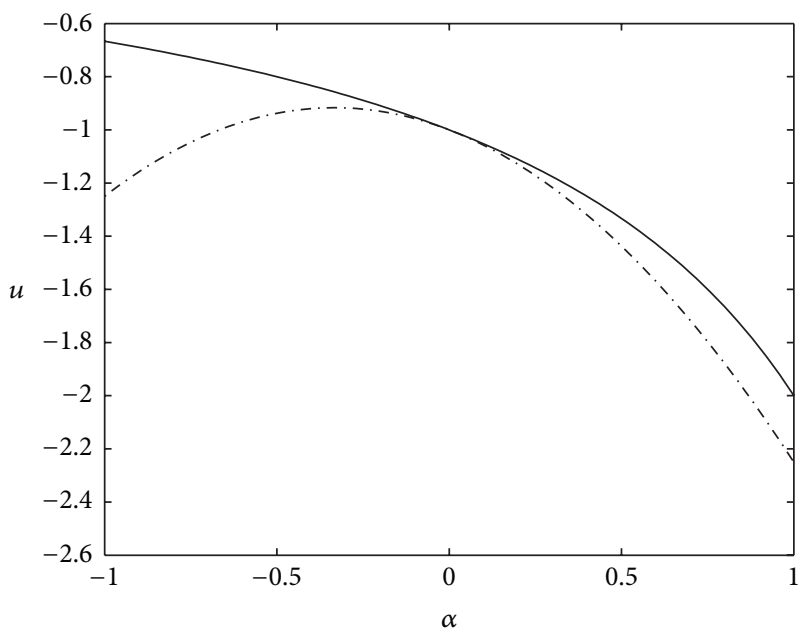

FIGURE 1: Influence of the nonlinear coefficient $\alpha(\varepsilon)$ on the difference between the new exact solution and the perturbation solution $[c=1, v=1$, and $\xi=0 ;-\cdot-$ : the perturbation solution; - : the new exact solution].

approaches positive and negative infinities, the corresponding tanh-coth solutions from (2) are

$$
\begin{aligned}
U_{\text {tanh-coth }}(\infty)=\frac{c}{\alpha}-\frac{c}{\alpha} \tanh \left(\frac{c}{2 v}(\infty)\right)=0, & \\
c>0 & >0 \\
U_{\text {tanh-coth }}(-\infty)=\frac{c}{\alpha}-\frac{c}{\alpha} \tanh \left(\frac{c}{2 v}(-\infty)\right)=\frac{2 c}{\alpha}, & \\
c>0 &
\end{aligned}
$$

The corresponding Cole-Hopf solutions from (18), at $x=$ $\pm \infty$, are

$$
\begin{gathered}
U_{\text {Cole-Hopf }}(\infty)=\frac{-2 \nu}{\alpha} \frac{k_{1} e^{k_{1}(\infty)+v k_{1}^{2} t}}{1+e^{k_{1}(\infty)+v k_{1}^{2} t}=\frac{-2 \nu k_{1}}{\alpha},} \quad k_{1}>0 ; \\
U_{\text {Cole-Hopf }}(-\infty)=\frac{-2 \nu}{\alpha} \frac{k_{1} e^{k_{1}(-\infty)+v k_{1}^{2} t}}{1+e^{k_{1}(-\infty)+v k_{1}^{2} t}}=0, \quad k_{1}>0 ; \\
U_{\text {Cole-Hopf }}(\infty)=\frac{-2 \nu}{\alpha} \frac{k_{1} e^{k_{1}(\infty)+v k_{1}^{2} t}}{1+e^{k_{1}(\infty)+v k_{1}^{2} t}}=0, \quad k_{1}<0 ; \\
U_{\text {Cole-Hopf }}(-\infty)=\frac{-2 \nu}{\alpha} \frac{k_{1} e^{k_{1}(-\infty)+v k_{1}^{2} t}}{1+e^{k_{1}(-\infty)+v k_{1}^{2} t}}=\frac{-2 \nu k_{1}}{\alpha}, \\
k_{1}<0 .
\end{gathered}
$$


TABle 1: Waves types of the tanh-coth solutions, the Cole-Hopf solution, and the new exact solution of the general Burgers' equation.

\begin{tabular}{cccccccc}
\hline \multicolumn{7}{l}{ Parameters } & \multicolumn{9}{c}{ Exact solutions } \\
\multicolumn{1}{c}{$c$} & \multicolumn{2}{c}{ The tanh-coth solutions } & The Cole-Hopf solution & The new exact solution \\
\hline$>0$ & $>0$ & Kink & Traveling wave & NA & NA & Kink & Traveling wave \\
$<0$ & $>0$ & Kink & Traveling wave & NA & NA & Kink & Kink \\
$>0$ & $<0$ & NA & NA & Traveling wave & Traveling wave & Kink & Traveling wave \\
$<0$ & $<0$ & NA & NA & Traveling wave & Traveling wave & Kink & Kink \\
\hline
\end{tabular}

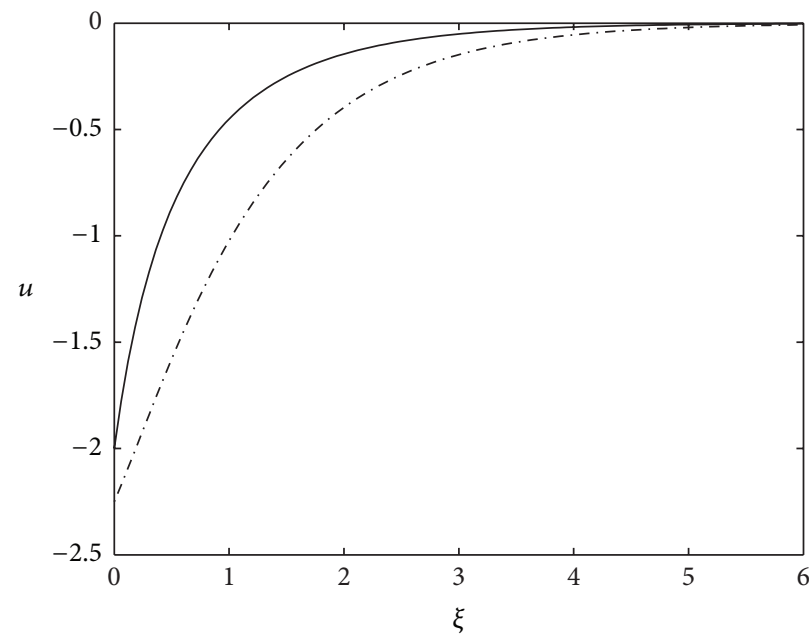

(a)

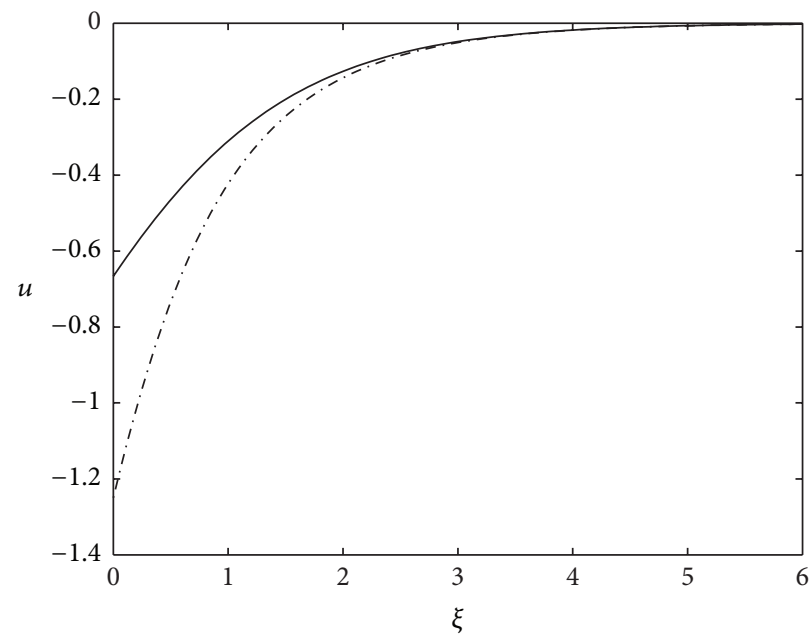

(b)

FiguRE 2: Difference between the new exact solution and the perturbation solution with various $\xi[\nu=1, c=1$, and (a) $\alpha=1$; (b) $\alpha=-1$; ---: the perturbation solution; - : the new exact solution].

The corresponding new exact solutions from (23), at $x= \pm \infty$, are

$$
\begin{gathered}
U_{\text {new }}(\infty)=\frac{-2 c}{2 v e^{(c / v)(\infty)}-\alpha}=0, \quad c>0 ; \\
U_{\text {new }}(-\infty)=\frac{-2 c}{2 v e^{(c / v)(-\infty)}-\alpha}=\frac{2 c}{\alpha}, \quad c>0 ; \\
U_{\text {new }}(\infty)=\frac{-2 c}{2 v e^{(c / v)(\infty)}-\alpha}=\frac{2 c}{\alpha}, \quad c<0 ; \\
U_{\text {new }}(-\infty)=\frac{-2 c}{2 v e^{(c / v)(-\infty)}-\alpha}=0, \quad c<0 .
\end{gathered}
$$

Hence, they satisfy the second condition of a kink wave. After numerical calculation using the software MATLAB, it can be found that the solutions of (1) with $\alpha<0$, (2), and (18) all satisfy the first condition of a kink wave. The kinds of waves of the tanh-coth solutions, (2)-(5), the Cole-Hopf solution, (18), and the new exact solution, (23), are summarized in Table 1.

In the following, the three different kinds of solutions, (2), (18), and (23), are examined and compared. As the nonlinear term coefficient of $\alpha$ varies from -1 to 1 , the difference between the new exact solution and the Cole-Hopf solution, (18), with $v=1, c=1, k_{1}=-1, x=1$, and $t=1$, is shown in Figure 3. It should be mentioned that under this case, the tanh-coth solution, (2), is the same as the Cole-Hopf solution. It can be found that the new exact solution is a continuous

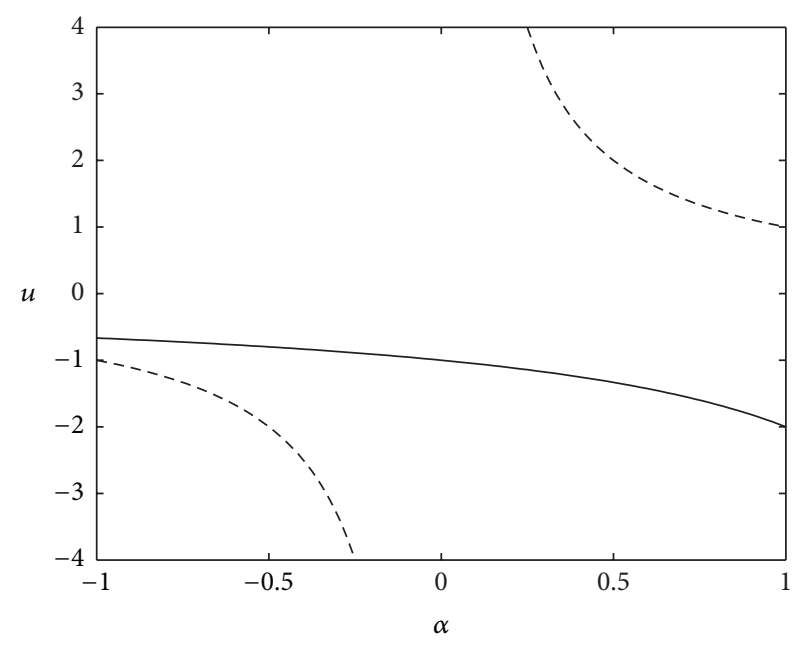

FIGURE 3: Comparison among the new exact solution, the tanh-coth solution, and the Cole-Hopf solution as the nonlinear coefficient $\alpha$ varies from -1 to $1\left[t=1, x=1, c=1, v=1\right.$, and $k_{1}=-1$; - : the new exact solution; --: the Cole-Hopf solution and the tanh-coth solution].

function of $\alpha$ while the other solution has discontinuity at $\alpha=0$. This phenomenon can be easily observed from the solution forms, namely, (2) and (18). When the nonlinear 


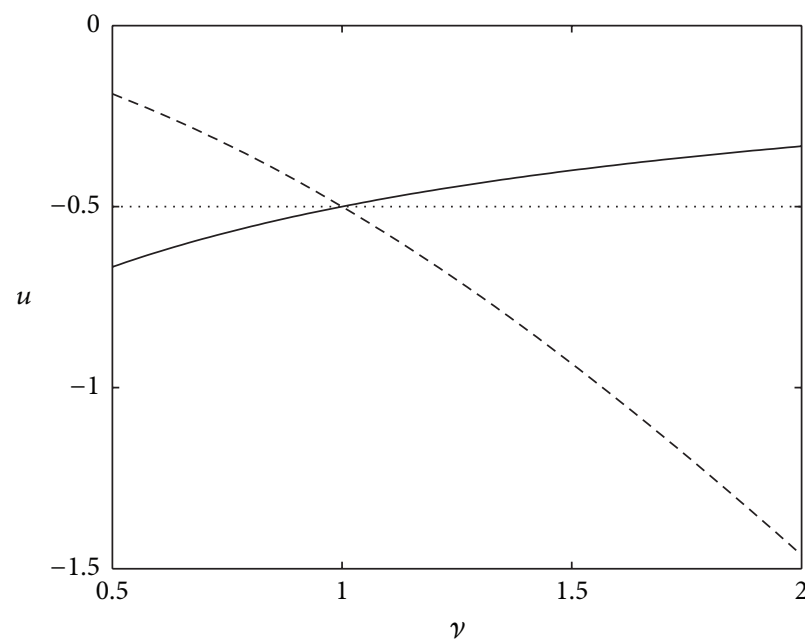

(a)

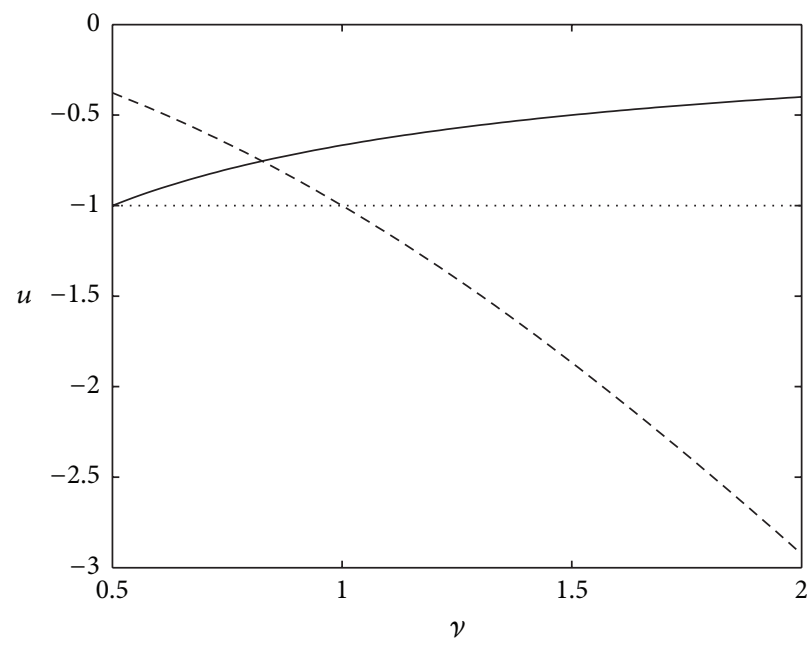

(b)

FIGURE 4: Influence of the kinematics viscosity coefficient $v$ on the three solutions $\left[x=1, c=1, t=1, k_{1}=-1\right.$, (a) $\alpha=-2$, and (b) $\alpha=-1$; -: the new exact solution; $\cdots$ : the tanh-coth solution; --: the Cole-Hopf solution].

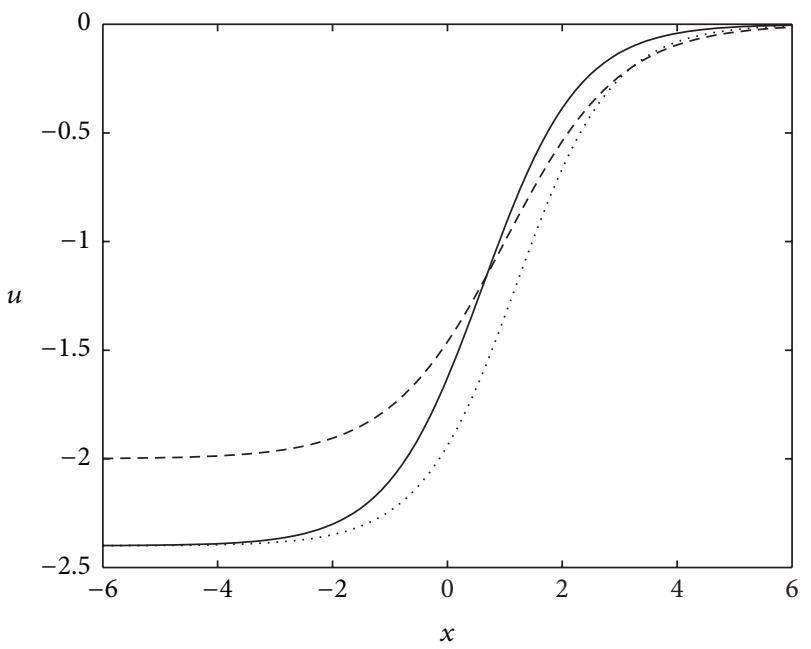

(a)

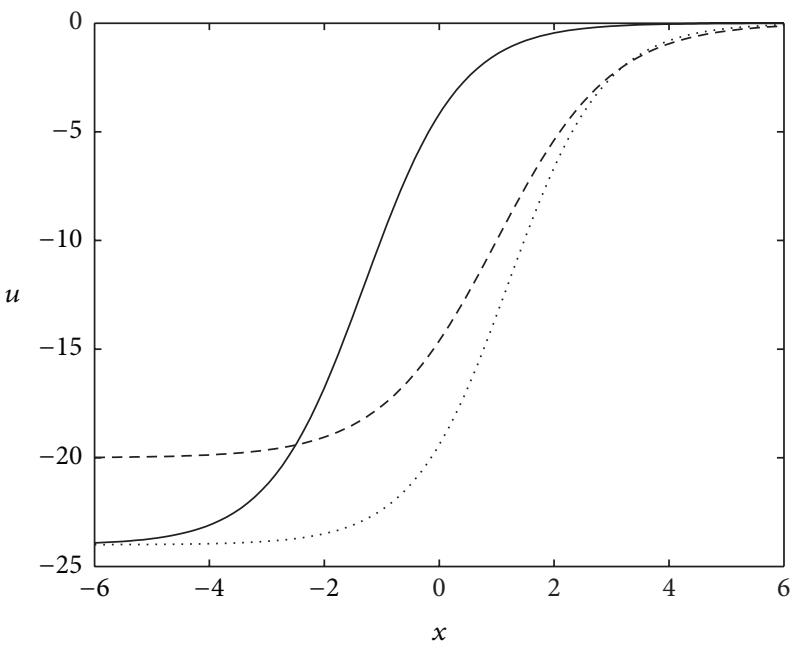

(b)

Figure 5: The new exact solution, the tanh-coth solution, and the Cole-Hopf solution with various $x$ and different nonlinear coefficients $\alpha$ $\left[c=1.2, t=1, v=1, k_{1}=-1\right.$, (a) $\alpha=-1$, and (b) $\alpha=-0.1$; - : the new exact solution; $\cdots$ : the tanh-coth solution; - -: the Cole-Hopf solution].

coefficient $\alpha$ in the tanh-coth solution and the Cole-Hopf solution is reduced to zeros, the solutions approach infinity.

Figure 4 shows the influence of the kinematics viscosity coefficient $v$ on the three solutions as $x=1, c=1, t=1$, $k_{1}=-1$, and $\alpha=-2$ or -1 . As can be seen, the coefficient $v$ has no influence on the tanh-coth solution. As the coefficient $v$ is increased, the new exact solution increases and the ColeHopf solution decreases, respectively. Under the unique case with $c=1, v=1, k_{1}=-1$, and $\alpha=-2$, the three different solutions are the same.

Figure 5 shows the new exact solution, the tanh-coth solution, and the Cole-Hopf solution with various $x$ and different negative nonlinear coefficients $\alpha$. It can be found that these three solutions are all kink solutions and are consistent with the summarized results in Table 1 . As mentioned in Section 2.3, with constants $x, c, t$, and $k_{1}$, the tanh-coth solution and the Cole-Hopf solution are both proportional to $1 / \alpha$ and become infinite as $\alpha$ approaches zero. The steep slope in the solutions is kept at the same location; however, this is not the case for the new exact solution. Under the same physical conditions, the new exact solution will not become infinite as $\alpha$ approaches zero. Moreover, the location of the steep slope in the new exact solution will slide and change as $\alpha$ is changed. As shown in Figure 5, the location of the steep 
slope in the new exact solution slides to the left as $\alpha$ is changed from -1 to -0.1 . We have named this peculiarity the kinksliding phenomenon.

\section{Conclusions}

In this paper, a new exact solution of the general Burgers' equation was derived by a direct integration method and the simplest equation method with the Bernoulli equation as the simplest equation. It was shown that the proposed derived exact solution can be successfully reduced to linearity, while the nonlinear term coefficient approaches zero. In addition, a general Cole-Hopf transformation solution was introduced. The differences among the newly derived exact solution, the other existing exact solutions, and the perturbation solution were elaborated. It was shown that when $\alpha=0$, both the new solution and perturbation solution lead to linearity and are the same; however, when the absolute value of $\alpha$ is increased to one, the perturbation solution has significant errors. With constants $x, c, t$, and $k_{1}$, the tanh-coth solutions and the ColeHopf solution are proportional to $1 / \alpha$ and become infinite as $\alpha$ approaches zero. Although the steep slope in the solutions is maintained at the same location, under the same physical conditions, the new exact solution will not become infinite as $\alpha$ approaches zero. Moreover, the location of the steep slope in the new exact solution slides, which was termed the kinksliding phenomenon. Extensions of the proposed methods to study different nonlinear differential equations without linearized solutions are expected in future works.

\section{Conflict of Interests}

The authors declare that there is no conflict of interests regarding the publication of this paper.

\section{Acknowledgment}

It is gratefully acknowledged that this work was supported by the Ministry of Science and Technology of Taiwan, under Grants NSC 102-2221-E-006-050 and MOST 103-2221-E-006048.

\section{References}

[1] H. Bateman, "Some recent researches on the motion of fluids," Monthly Weather Review, vol. 43, no. 4, pp. 163-170, 1915.

[2] J. M. Burgers, "A mathematical model illustrating the theory of turbulence," Advances in Applied Mechanics, vol. 1, pp. 171-199, 1948.

[3] N. Sugimoto, "Burgers equation with a fractional derivative; hereditary effects on nonlinear acoustic waves," Journal of Fluid Mechanics, vol. 225, pp. 631-653, 1991.

[4] E. Weinan, K. Khanin, A. Mazel, and Y. Sinai, "Invariant measures for Burgers equation with stochastic forcing," Annals of Mathematics, vol. 151, no. 3, pp. 877-960, 2000.

[5] G. B. Whitham, Linear and Nonlinear Waves, vol. 42 of Pure and Applied Mathematics, John Wiley \& Sons, 2011.

[6] G. W. Bluman and J. D. Cole, "The general similarity solution of the heat equation," Journal of Mathematics and Mechanics, vol. 18, pp. 1025-1042, 1969.
[7] J. Bec and K. Khanin, "Burgers turbulence," Physics Reports, vol. 447, no. 1-2, pp. 1-66, 2007.

[8] J. B. Bell, P. Colella, and H. M. Glaz, "A second-order projection method for the incompressible navier-stokes equations," Journal of Computational Physics, vol. 85, no. 2, pp. 257-283, 1989.

[9] A. A. Soliman, "The modified extended tanh-function method for solving Burgers-type equations," Physica A: Statistical Mechanics and Its Applications, vol. 361, no. 2, pp. 394-404, 2006.

[10] A. Ebaid, "Exact solitary wave solutions for some nonlinear evolution equations via Exp-function method," Physics Letters A, vol. 365, no. 3, pp. 213-219, 2007.

[11] A.-M. Wazwaz, "Multiple-front solutions for the Burgers equation and the coupled Burgers equations," Applied Mathematics and Computation, vol. 190, no. 2, pp. 1198-1206, 2007.

[12] A. M. Wazwaz, Partial Differential Equations and Solitary Waves Theory, Springer, 2010.

[13] E. Hopf, "The partial differential equation $u_{t}+u u_{x}=u_{x x}$ " Communications on Pure and Applied Mathematics, vol. 3, pp. 201-230, 1950.

[14] J. D. Cole, "On a quasi-linear parabolic equation occurring in aerodynamics," Quarterly of Applied Mathematics, vol. 9, pp. 225-236, 1951.

[15] T. Ohwada, "Cole-Hopf transformation as numerical tool for the burgers equation," Applied and Computational Mathematics, vol. 8, no. 1, pp. 107-113, 2009.

[16] B. M. Vaganan, "Cole-Hopf transformations for higher dimensional Burgers equations with variable coefficients," Studies in Applied Mathematics, vol. 129, no. 3, pp. 300-308, 2012.

[17] N. A. Kudryashov, "Seven common errors in finding exact solutions of nonlinear differential equations," Communications in Nonlinear Science and Numerical Simulation, vol. 14, no. 9-10, pp. 3507-3529, 2009.

[18] N. A. Kudryashov, "One method for finding exact solutions of nonlinear differential equations," Communications in Nonlinear Science and Numerical Simulation, vol. 17, no. 6, pp. 2248-2253, 2012.

[19] M. R. Spiegel, L. Seymour, and L. John, Schaum's Outlines Mathematical Handbook of Formulas and Tables, Schaum Publications, 1968

[20] N. K. Vitanov, "Application of simplest equations of Bernoulli and Riccati kind for obtaining exact traveling-wave solutions for a class of PDEs with polynomial nonlinearity," Communications in Nonlinear Science and Numerical Simulation, vol. 15, no. 8, pp. 2050-2060, 2010.

[21] N. K. Vitanov, "Modified method of simplest equation: powerful tool for obtaining exact and approximate traveling-wave solutions of nonlinear PDEs," Communications in Nonlinear Science and Numerical Simulation, vol. 16, no. 3, pp. 1176-1185, 2011.

[22] N. K. Vitanov, "On modified method of simplest equation for obtaining exact and approximate solutions of nonlinear PDEs: the role of the simplest equation," Communications in Nonlinear Science and Numerical Simulation, vol. 16, no. 11, pp. 4215-4231, 2011. 


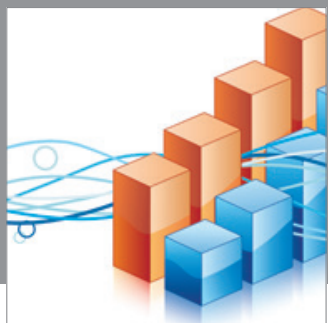

Advances in

Operations Research

mansans

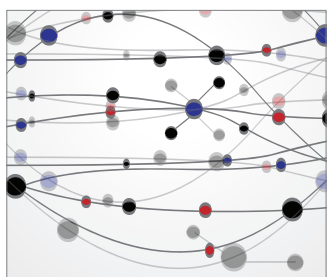

The Scientific World Journal
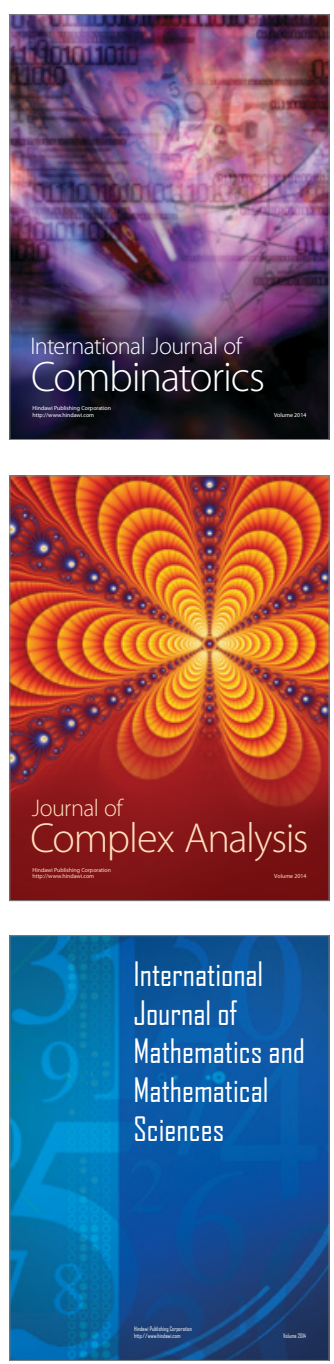
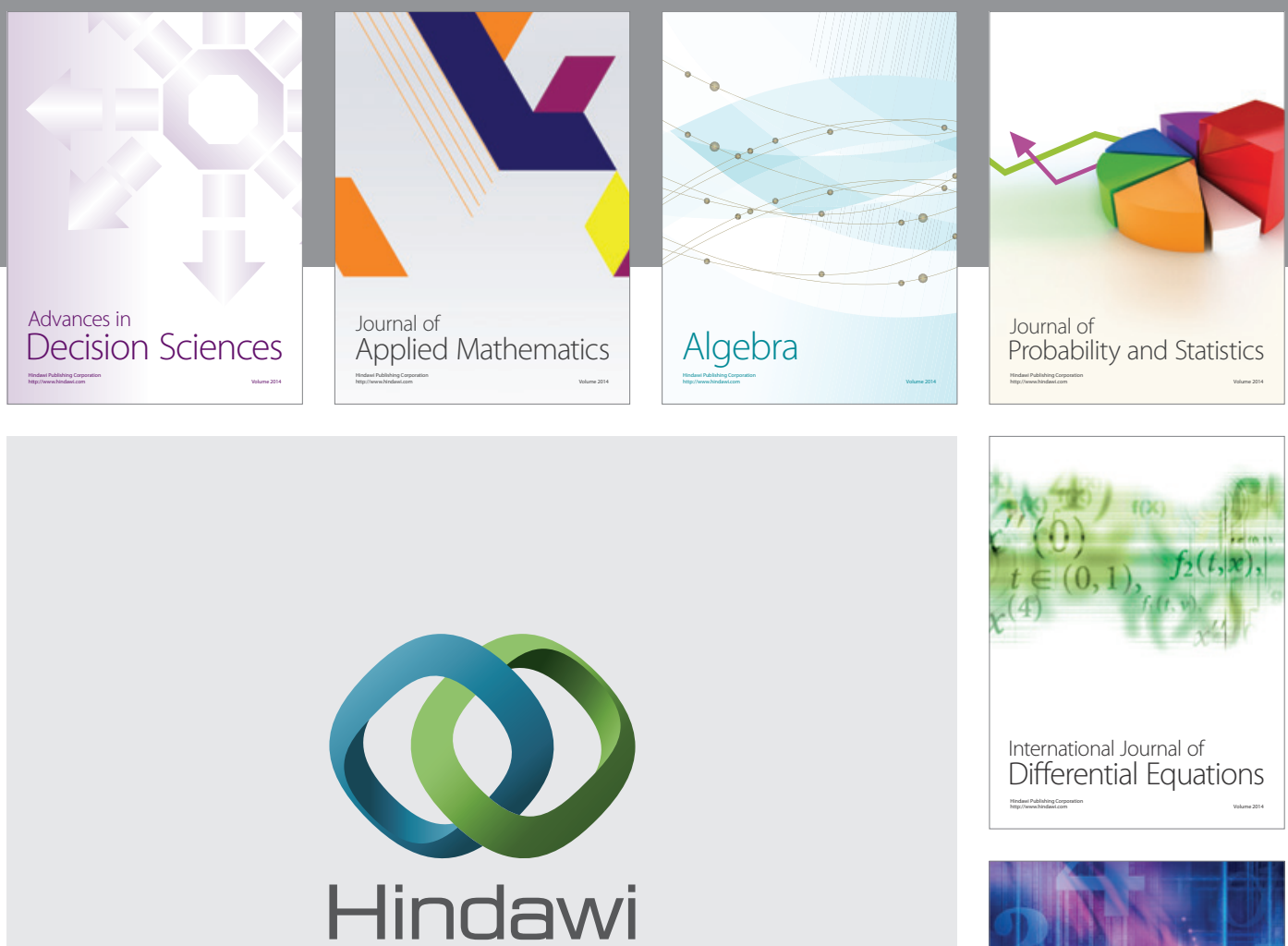

Submit your manuscripts at http://www.hindawi.com
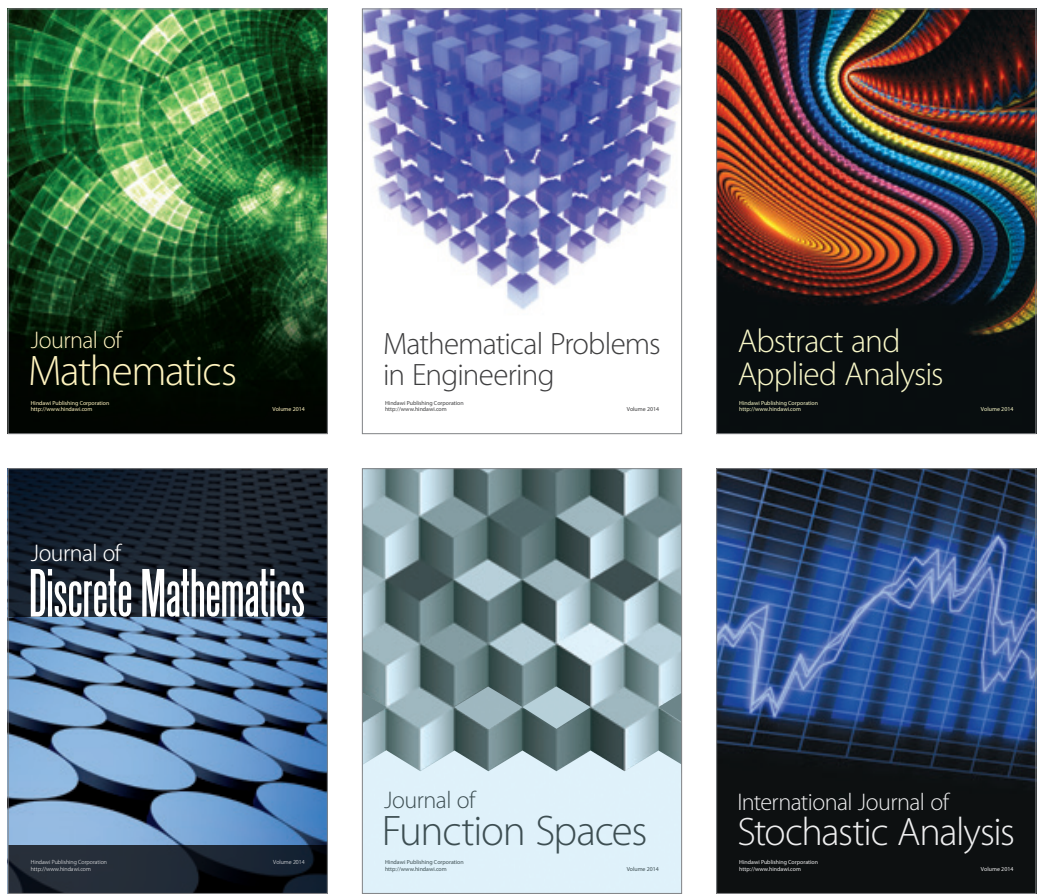

Journal of

Function Spaces

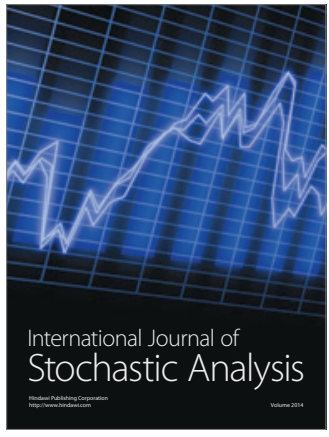

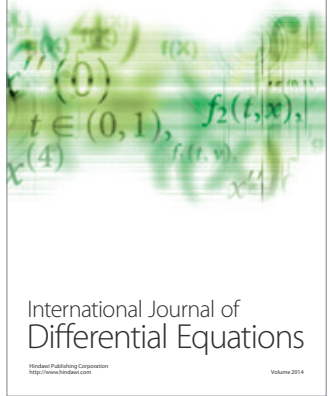
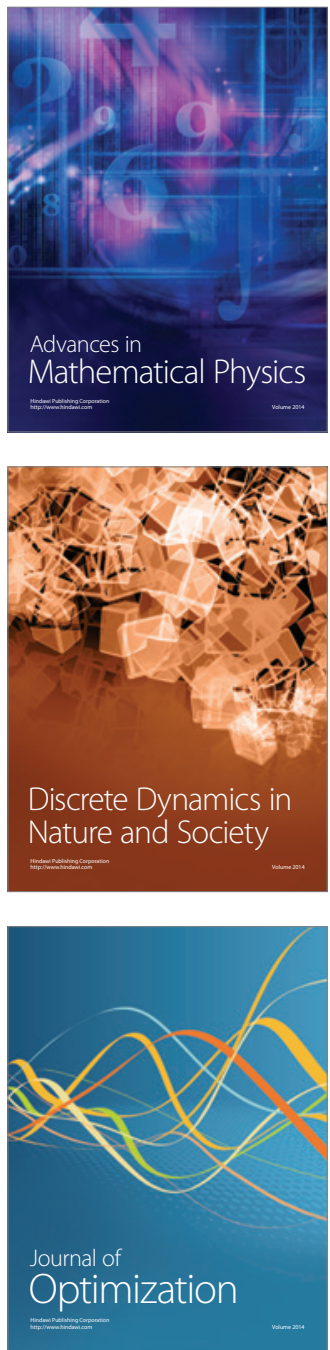